Agrotrópica 31(3): 219-224. 2019.

Centro de Pesquisas do Cacau, Ilhéus, Bahia, Brasil

\title{
ESCARABEÍDEOS (COLEOPTERA: SCARABAEIDAE) COLETADOS EM ARMADILHAS PITFALL EM UM REMANESCENTE FLORESTAL NO MUNICÍPIO DE PLÁCIDO DE CASTRO, AC
}

\author{
Rodrigo Souza Santos ${ }^{1 *}$, Clemeson Silva de Souza ${ }^{2}$, Conceição Paula Bandeira Rufino ${ }^{3}$ \\ 1Embrapa Acre, Rodovia BR 364, km 14, CP 321, 69900-970, Rio Branco, AC, Brasil. ²Programa de Pós-Graduação em \\ Produção Vegetal, Universidade Federal do Acre (UFAC), 69920-900, Rio Branco-AC, Brasil. \\ ${ }^{3}$ Programa de Pós-Graduação em Ciência, Inovação e Tecnologia para Amazônia, Universidade Federal do Acre (UFAC), \\ 69920-900, Rio Branco-AC, Brasil.
}

*Autor para correspondência: rodrigo.s.santos@embrapa.br

\begin{abstract}
Muitos processos que estruturam o solo são realizados por invertebrados edáficos, sendo os insetos o principal grupo. A ordem Coleoptera é a maior dentro da classe Insecta, tendo a família Scarabaeidae uma das mais representativas e importantes. O objetivo deste trabalho foi conhecer a fauna de Scarabaeidae edáficos em um remanescente florestal no município de Plácido de Castro, AC. O estudo foi realizado entre agosto de 2014 a agosto de 2015, utilizando 22 armadilhas pitfalls (copos plásticos de $500 \mathrm{~mL}$ ) dispostas em dois transectos, distanciadas cinco metros entre si, com espaçamento de $10 \mathrm{~m}$ entre armadilhas. Em cada armadilha foi adicionada uma solução de formaldeído (1\%) e gotas de detergente neutro, com coletas quinzenais. No laboratório de Entomologia da Embrapa Acre os insetos foram triados e identificados com auxílio de literatura apropriada. Foram capturados 218 espécimes, representados por quatro tribos, sete gêneros e 15 espécies. A tribo Coprini foi a mais representativa, com nove espécies capturadas, sendo sete correspondendo ao gênero Canthidium. Pelo número de espécies capturadas e nichos alimentares conclui-se que o remanescente florestal estudado apresenta baixo grau de antropização.
\end{abstract}

Palavras-chave: Fauna edáfica, floresta tropical, Scarabaeoidea.

Dung beetles (Coleoptera: Scarabaeidae) collected in pitfall traps in a forest
emnant in the municipality of Plácido de Castro, Acre state, Brazil. Many processes that structure the soil are carried out by edaphic invertebrates, with insects being the main group. The order Coleoptera is the largest within the class Insecta, with the family Scarabaeidae one of the most representative and important. The aim of this work was to know the edaphic fauna of Scarabaeidae in a forest remnant in the municipality of Plácido de Castro, Acre state, Brazil. The study was realized between August 2014 to August 2015, using 22 pitfall traps (500 mL plastic cups) arranged in two transects, five meters apart, with $10 \mathrm{~m}$ spacing between traps. In each trap was added a solution of formaldehyde $(1 \%)$ and neutral detergent drops, with biweekly samplings. In the Embrapa Acre's Entomology Laboratory the insects were tried and identified with the aid of appropriate literature. Twenty-one specimens, represented by four tribes, seven genera and 15 species were captured. The Coprini tribe was the most representative, with nine species captured, of which seven corresponded to the genus Canthidium. By the number of species captured and food niches, it can be concluded that the studied forest remnant presents a low degree of anthropization.

Key words: Edaphic fauna, tropical forest, Scarabaeoidea. 


\section{Introdução}

Áreas florestais atenuam a erosão e compactação do solo pela redução do impacto da pluviosidade e os dosséis das árvores configuram uma barreira que reduz a velocidade dos ventos (Castro et al., 1996). O solo desempenha um papel importante na preservação do nível dos rios, fontes de água, além de promover um armazenamento e filtragem de elementos químicos tóxicos (Nascimento et al., 2004). Os fragmentos florestais são geralmente de tamanhos diferentes e coabitam com áreas usadas para fins pecuários, agrícolas e/ou florestais (Aleixo, 1999). As modificações estruturais da vegetação influenciam diretamente na composição da fauna local (Doube e Wardhalgh, 1991; Laurino et al., 2006).

Dentre os invertebrados, os insetos são considerados bons bioindicadores, devido ao alto número de espécies existentes, facilidade de captura, habitats que colonizam e sensibilidade às condições ambientais. Ademais, os insetos participam ativamente nos processos biológicos dos ecossistemas onde se encontram (Favero et al., 2011). Como exemplo de insetos bioindicadores, destacam-se os representantes da ordem Coleoptera, pela facilidade de respostas às alterações antrópicas, como a fragmentação florestal (Aquino, 2001). Cada organismo bioindicador pode responder de maneira diferente de acordo com a ocorrência e graus de perturbações ambientais, revelando informações sobre um determinado distúrbio (Büchs, 2003).

A ocorrência e distribuição espacial de artrópodes edáficos podem ser avaliadas, com emprego de armadilhas de queda (pitfall trap) em transectos, dispostas em seus habitats. O emprego deste tipo de armadilha permite determinar a agregação e distribuição espacial de populações, contribuindo para o entendimento da imigração ou emigração de organismos edáficos entre áreas, em resposta às alterações ambientais antrópicas (Bedford e Usher, 1994).

A ordem Coleoptera abriga insetos comumente encontrados na fauna edáfica de ambientes florestais e, àqueles pertencentes à família Scarabaeidae, são um dos grupos mais representativos e diversos desta ordem. Esta família apresenta majoritariamente espécies de hábito detritívoro, as quais se alimentam de carcaças, frutos em decomposição e fezes de vertebrados (Nichols et al., 2008; Slade et al., 2011). Essas características tornam esta família um grupo importante tanto para estudos entomológicos (Bai et al., 2015), quanto para entender o processo de ciclagem de nutrientes através da decomposição de matéria orgânica e medir graus de antropização, pela presença ou ausência de determinadas espécies (Wink et al., 2005; Silva e Hernández, 2016).

Estudos sobre a fauna de escarabeídeos ainda são escassos na Amazônia brasileira, principalmente pela sua extensão territorial e diversidade de habitats (Klein, 1989; Vaz-de-Mello, 1999). Trabalhos envolvendo escarabeídeos amazônicos são reportados para os estados do: Pará (Andresen, 2002; Scheffler, 2005; Matavelli e Louzada, 2008; Barlow et al., 2010), Amazonas (Quintero e Roslin, 2005; Radtke et al., 2007; Quintero \& Halffter, 2009; Korasaki et al., 2012), Rondônia (Vulinec, 2000; Silva et al., 2014) e Acre (Vaz-de-Mello, 1999; Santos et al., 2018).

Diante da relevância dos Scarabaeidae para os ecossistemas, aliado à escassez de estudos sobre este grupo de insetos no estado do Acre, o presente trabalho objetivou conhecer a fauna de escarabeídeos, em um remanescente florestal localizado no município de Plácido de Castro, AC.

\section{Material e Métodos}

O estudo foi realizado entre agosto de 2014 a agosto de 2015, em um remanescente de floresta primária, de aproximadamente oito hectares, inserido na fazenda Amoty, localizada no município de Plácido de Castro, AC $\left(10^{\circ} 20^{\prime} 19.3^{\prime \prime} \mathrm{S} ; \quad 6^{\circ} 29^{\prime} 06.2^{\prime \prime} \mathrm{W}\right)$. Esse remanescente encontra-se bordeado por vegetação de capoeira e pastagem. A vegetação de capoeira corresponde à floresta nos estágios de inicial a médio de regeneração (IBGE, 2012).

O clima da região é quente e úmido, com temperaturas médias anuais variando de 24,5 a $32^{\circ} \mathrm{C}$ e, tipo Aw (tropical de savana), com duas estações distintas: uma seca e uma chuvosa, de acordo com a classificação de Köppen. A estação seca inicia-se no mês de maio estendendo-se até o mês de outubro, com início do período chuvoso (Oliveira e Braz, 1998).

O levantamento da população de escarabeídeos foi realizado com uso de armadilhas de solo (tipo pitfall), sem utilização de atrativos alimentares, conforme 
metodologia adaptada de Cajaiba e Silva (2015). As armadilhas foram espaçadas $10 \mathrm{~m}$ entre si e dispostas em dois transectos (11 armadilhas por transecto), da bordadura ao interior do remanescente. Os transectos foram espaçados cinco metros entre si.

As armadilhas foram confeccionadas com copos plásticos de capacidade de $500 \mathrm{~mL}$ (nove centímetros de diâmetro), contendo uma solução de formaldeído (1\%) e algumas gotas de detergente neutro (Brennan et al., 1999; Martins e Lise, 2009). Em cada armadilha foi instalada uma cobertura de madeira, a fim de protegê-las do transbordo pela água da chuva e/ou entupimento por folhas ou detritos. A altura dessas coberturas foi suficiente para não inferir no acesso dos besouros às armadilhas.

Quinzenalmente procedia-se o recolhimento e substituição das armadilhas, totalizando 22 amostragens durante o ensaio. As amostras foram levadas ao Laboratório de Entomologia da Embrapa Acre, onde foi realizada a triagem e análise sob estereomicroscópio. Com auxílio de peneira granulométrica, o conteúdo de pitfall era vertido e triado, separando os escarabeídeos do restante do material, o qual foi descartado. Ao término de cada triagem os espécimes eram acondicionados em frascos identificados contendo álcool (70\%), e enviados ao Dr. Paschoal Coelho Grossi (Universidade Federal Rural de Pernambuco (UFRPE), Recife, PE), para identificação. Os insetos foram depositados na Coleção Entomológica da Universidade Federal Rural de Pernambuco - CERPE.

\section{Resultados e Discussão}

Foram coleados 218 espécimes de escarabeídeos no remanescente florestal durante o período de estudo, distribuídos em quatro tribos, sete gêneros e 15 espécies. A tribo Coprini foi a que apresentou maior diversidade (nove espécies), seguida por Canthonini, Phanaeini e Oniticellini (Tabela 1).

As espécies Canthidium sp. 4 (55 espécimes), Canthidium sp.7 (41 espécimes) e Canthon aff. luctuosus (33 espécimes) foram as mais abundantes, correspondendo a 25,2, 19,0 e 15,1\% respectivamente, de todos os coleópteros coletados. Em contrapartida, as espécies Eurysternus caribaeus $(0,4 \%)$ e Dichotomius aff. boreus $(0,4 \%)$ foram as menos frequentes, sendo registrado apenas um espécime de cada espécie (Tabela 1).

Apesar de ser um dos gêneros mais especiosos dentro de Scarabaeidae, Canthidium é um dos menos estudados (Martínez \& Halffter, 1986a; 1986b). Uma revisão taxonômica é fortemente necessária para este gênero, o qual atualmente possui vários subgêneros. Espécies de Canthidium são profusas em ambientes florestais (Durães et al., 2005), principalmente devido à diversidade de hábitos alimentares encontradas, com espécies coprófagas, frugívoras, carpófagas, micófagas e necrófagas (Vaz-de-Mello, 1999; Falqueto et al., 2005). A maioria da espécies de Canthidium transportam o recurso alimentar para túneis escavados abaixo do depósito de alimento (Vaz-de-Mello et al., 2011; Arias-Buriticá e Vaz-de-Mello, 2012)

Um número significativo de espécimes de Canthidium foi capturado por Durães et al. (2005), em levantamento utilizando armadilhas pitfall iscadas (com atrativos alimentares), em região de transição de Cerrado e Floresta Atlântica em Minas Gerais.

Um estudo conduzido por Vaz-de-Mello (1999) no estado do Acre, em floresta secundária loalizada no Parque Zoobotânico, registrou 36 espécies de escarabeídeos (seis espécies de Canthidium). Foram empregadas nesse estudo, além de armadilhas pitfall iscadas, armadilha Malaise e coleta manual. Em trabalho similar, realizado por Santos et al. (2018) em Rio Branco, AC, o gênero Canthidium também foi um dos mais capturados em armadilhas pitfalls com atrativos alimentares, sendo o coração bovino em decomposição o principal atrativo. Estes estudos demonstram que Canthidium é um gênero comum e possui ampla distribuição geográfica no Brasil.

Os gêneros Coprophanaeus, Oxysternon, Deltochilum, Canthon e Dichotomius englobam espécies com os mais variados hábitos alimentares, mas, a grande maioria apresenta hábito necrófago ou copro-necrófago, vivendo principalmente de matéria orgânica em decomposição (Vaz-de-Mello, 1999; Korasaki et al., 2012). Assim, apesar de o presente estudo não ter utilizado armadilhas com atrativos alimentares, a grande maioria das espécies capturadas têm hábitos copro-necrófagos. Uma hipótese levantada por Favero et al. (2011) para tal fato, seria pelas fezes deixadas por animais presentes em ambientes florestais, os quais consomem frutos 
Tabela 1. Espécies de escarabeídeos coletados em armadilhas pitfall, em remanescente florestal no município de Plácido de Castro, AC, no período de agosto de 2014 a agosto de 2015

\begin{tabular}{|c|c|c|c|}
\hline Espécie & Tribo & Frequência absoluta & Frequência relativa (\%) \\
\hline Coprophanaeus telamon (Erichson) & Phanaeini & 11 & 5,0 \\
\hline Oxysternon silenus Castelnau & & 10 & 4,6 \\
\hline Eurysternus caribaeus (Herbst) & Oniticellini & 01 & 0,4 \\
\hline Deltochilum schefflerorum Silva, Louzada e Vaz-de-Mello & Canthonini & 14 & 6,4 \\
\hline Deltochilum aff. irroratum & & 13 & 6,0 \\
\hline Canthon aff. luctuosus & & 33 & 15,1 \\
\hline Dichotomius sp. & Coprini & 09 & 4,2 \\
\hline Dichotomius aff. boreus & & 01 & 0,4 \\
\hline Canthidium sp. 1 & & 03 & 1,4 \\
\hline Canthidium sp. 2 & & 04 & 1,8 \\
\hline Canthidium sp. 3 & & 07 & 3,2 \\
\hline Canthidium sp. 4 & & 55 & 25,2 \\
\hline Canthidium sp. 5 & & 11 & 5,0 \\
\hline Canthidium sp. 6 & & 05 & 2,3 \\
\hline Canthidium sp. 7 & & 41 & 19,0 \\
\hline Total & & 218 & 100 \\
\hline
\end{tabular}

produzidos pelas plantas e defecam no local, atraindo organismos coprófagos.

Os escarabeídeos são habilidosos removedores de fezes, principalmente em ambientes pastoris (RidsdillSmith \& Hayles, 1990), contribuindo para o controle biológico de moscas hematófagas. Ademais, besouros escarabeídeos podem ser utilizados como bioindicadores em ecossistemas de florestas tropicais (Halffter \& Favila, 1993; Wink et al., 2005), o que torna esse grupo especialmente importante em estudos de monitoramento e impacto ambiental.

Atualmente o estado do Acre conta com 43 espécies de Scarabaeidae registradas (Vaz-de-Mello, 2000; Santos et al., 2018). Para efeito de comparação, o estado de São Paulo (Estado mais bem amostrado do Brasil) possui 192 espécies registradas possuindo uma área de floresta preservada muito inferior, em comparação ao estado do Acre. Apesar do presente estudo não relatar nenhum novo registro de escarabeídeo para o estado do Acre, há grande probabilidade de novos registros e/ ou novas espécies serem reportados, visto a extensa área florestal intacta ainda não inventariada no Estado.

O remanescente florestal estudado vem sofrendo com derrubada e queima da floresta, com intuito de ampliar a área de pastagem para incremento da atividade pecuária em Plácido de Castro. Entretanto, foi verificada uma rica fauna de escarabeídeos nessa área, com capacidade de realizar a ciclagem da matéria orgânica. Dessa forma, é fortemente recomendada a realização de novos levantamentos desse grupo de insetos em outros municípios do Estado.

\section{Conclusão}

Pelo número de espécies de escarabeídeos capturada nesse levantamento e pelos seus nichos alimentares, conclui-se que o remanescente florestal estudado tem baixo grau de antropização.

\section{Literatura Citada}

ALEIXO, A. 1999. Effects of a selective logging on a bird community in the Brazilian Atlantic Forest. The Condor 101:537-548.

ANDRESEN, E. 2002. Dung beetles in a Central Amazonian rainforest and their ecological role as secondary seed dispersers. Ecological Entomology 27(3):257-270.

AQUINO, A. M. de. 2001. Manual para coleta de macrofauna do solo. Seropédica, Embrapa Agrobiologia. Embrapa-CNPAB, Documentos, 130.21p. 
ARIAS-BURITICÁ, J. A.; VAZ-DE-MELLO, F. Z. 2012. Redescripción de Dichotomius camposeabrai y D. nemoricola (Coleoptera: Scarabaeidae: Scarabaeinae), con apuntes sobre su posición sistemática. Revista Mexicana de Biodiversidad 83(2):387-395.

BARLOW, J. et al. 2010. Improving the design and management of forest strips in human-dominated tropical landscapes: a field test on Amazonian dung beetles. Journal of Applied Ecology 47(4):779-788.

BAI, M. et al. 2015. Mandible evolution in the Scarabaeinae (Coleoptera: Scarabaeidae) and adaptations to coprophagous habits. Frontiers in Zoology 12(30):1-10.

BEDFORD, S. E.; USHER, M. B. 1994. Distribution of arthropod species across the margins of farm woodlands. Agricuture, Ecosystems and Environment 48(3):295-305.

BRENNAN, K. E. C.; MAJER, J. D.; REYGAERT, N. 1999. Determination of an optimal pitfall trap size for sampling spiders in a Western Australian Jarrah forest. Journal of Insect Conservation 3(4): 297-307.

BÜCHS, W. 2003. Biodiversity and agri-environmental indicators-general scopes and skills with special reference to the habitat level. Agricuture, Ecosystems and Environment 98(1-3):35-78.

CAJAIBA, R. L.; SILVA, W. B. da. 2015. Abundância e diversidade de Coleoptera (Arthropoda: Insecta) de solo em Fragmentos de Capoeira ao entorno da zona urbana do município de Uruará-PA, Brasil. EntomoBrasils 8(1):30-37.

CASTRO, C. R. T.; LEITE, H. G.; COUTRO, L. 1996. Sistemas silvipastoris no Brasil: Potencialidades e entraves. Revista Árvore (Brasil) 20(4):575-582.

DOUBE, B. M.; WARDHALGH, K. G. 1991. Habitat associations and niche partitioning in an island dung beetle community. Acta Oecologica 12(4):451-459.

DURÃES, R.; MARTINS, W. P.; VAZ-DE-MELLO, F. Z.. 2005. Dung beetle (Coleoptera: Scarabaeidae) assemblages across a natural forest-cerrado ecotone in Minas Gerais, Brazil. Neotropical Entomology 34(5):721-731.
FALQUETO, S. A.; VAZ-DE-MELLO, F. Z.; SCHOEREDER, J. H. 2005. Are fungivorous Scarabaeidae less specialist? Ecología Austral 15: 17-22.

FAVERO, S.; SOUZA, H. A.; OLIVEIRA, A. K. M. 2011. Coleoptera (Insecta) as forest fragmentation indicators in the Rio Negro sub-region of the Pantanal, Mato Grosso do Sul, Brazil. Brazilian Journal of Biology 71(2):291-295.

HALFFTER, G.; FAVILA, M. E. 1993. The Scarabaeinae (Insecta: Coleoptera) an animal group for analyzing, inventorying and monitoring biodiversity in tropical rainforest and modified landscapes. Biology International 27:15-21.

INSTITUTO BRASILEIRO DE GEOGRAFIA E ESTATÍSTICA - IBGE. 2012. Manuais técnicos em geociências número 1. Manual técnico da vegetação brasileira. 2ed. Rio de Janeiro, RJ, IBGE. 271p.

KLEIN, B. C. 1989. Effects of forest fragmentation on dung and carrion beetle communities in Central Amazonia. Ecology 70(6):1715-1725.

KORASAKI, V. et al. 2012. Taxocenose de Scarabaeinae (Coleoptera: Scarabaeidae) em Benjamin Constant, AM. Acta Amazonica (Brasil) 42(3):423-432.

LAURINO, M. C. et al. 2006. Global meliponiculture: challenges and opportunities. Apidologie 37(2): 275-292.

MARTINEZ, A.; HALFFTER, G. 1986a. Two new species of Canthidium (Coleoptera, Scarabaeidae). Anales de la Escuela Nacional de Ciencias Biologicas Mexico 30(1-4):19-26.

MARTINEZ, A.; HALFFTER, G. 1986b. Status of the genus Canthidium Erichson (Coleoptera: Scarabaeidae: Scarabaeinae). Acta Zoológica Mexicana 18:19-40.

MARTINS, M.; LISE, A. 2009. As aranhas. In: Lisboa, P. L. B. (Org.). Caxiuanã: desafios para conservação de uma floresta nacional na Amazônia. Belém, PA, Museu Paraense Emílio Goeldi. pp. 381-388.

MATAVELLI, R. A.; LOUZADA, J. N. C. 2008. Invasão de áreas de savana intra-amazônicas por Digitonthophagus gazela (Fabricius, 1787) 
(Insecta: Coleoptera: Scarabaeidae). Acta Amazonica (Brasil) 38(1):153-158.

NASCIMENTO, P. C.; GIASSON, E.; INDA JÚNIOR, A. V. 2004. Aptidão de uso de solo e meio ambiente. In: Azevedo, A. C.; Dalmolin, R. S. D.; Pedron, F. de A. (eds.). Santa Maria, Solos \& Ambiente - I Fórum. pp.41-57.

NICHOLS, E. et al. 2008. Ecological functions and ecosystem services provided by Scarabaeinae dung beetle. Biological Conservation 141(6):1461-1474.

OLIVEIRA, M. V. N.; BRAZ, M. 1998. Manejo florestal em regime de rendimento sustentado aplicado à floresta do Campo Experimental da Embrapa/CPAF/AC. Boletim de Pesquisa, 21. Rio Branco, AC, Embrapa Acre. 45p.

QUINTERO, I.; HALFFTER, G. 2009. Temporal changes in a community of dung beetles (Insecta: Coleoptera: Scarabaeinae) resulting from the modification and fragmentation of tropical rain forest. Acta Zoológica Mexicana 25(3):625-649.

QUINTERO, I.; ROSLIN, T. 2005. Rapid recovery of dung beetle communities following habitat fragmentation in Central Amazonia. Ecology 86(12):3303-3311.

RADTKE, M. G.; FONSECA, C. R. V.; WILLIAMSON, G. B. 2007. The old and young Amazon: dung beetle biomass, abundance, and species diversity. Biotropica 39(6):725-730.

RIDSDILL-SMITH, T. J.; HAYLES, L. 1990. Stages of bush fly Musca vetustissima Walker (Diptera, Muscidae) killed by scarabaeinae dung beetles (Coleoptera, Scarabaeidae) in unfavourable cattle dung. Bulletin of Entomological Research 80(4): 473-478.

SANTOS, R. S.; SUTIL, W. P.; OLIVEIRA, J. F. A. de. 2018. Besouros escarabeídeos (Coleoptera: Scarabaeidae) coletados em remanescente florestal em Rio Branco, Acre, Brasil. Agrotropica (Brasil) 30(1):43-48.

SCHEFFLER, P. Y. 2005. Dung beetle (Coleoptera:
Scarabaeidae) diversity and community structure across three disturbance regimes in eastern Amazonia. Journal of Tropical Ecology 21(1): 9-19.

SILVA, R. J. et al. 2014. Rola-bostas (Coleoptera: Scarabaeidae: Scarabaeinae) de florestas e pastagens no sudoeste da Amazônia brasileira: Levantamento de espécies e guildas alimentares. Acta Amazonica (Brasil) 44(3):345-352.

SILVA, P. G. da; HERNÁNDEZ, M. I. M. 2016. Spatial variation of dung beetle assemblages associated with forest structure in remnants of southern Brazilian Atlantic Forest. Revista Brasileira de Entomologia 60(1):73-78.

SLADE, E. M.; MANN, D. J.; LEWIS, O. T. 2011. Biodiversity and ecosystem function of tropical forest dung beetles under contrasting logging regimes. Biological Conservation 144(1):166-174.

VAZ-DE-MELLO, F. Z. 1999. Scarabaeidae s. str. (Coleoptera: Scarabaeoidea) de um fragmento de floresta Amazônica no estado do Acre, Brasil. 1. Taxocenose. Anais da Sociedade Entomológica do Brasil (Brasil) 28(3):439-446.

VAZ-DE-MELLO, F. Z. 2000. Estado atual de conhecimento dos Scarabaeidae s. str. (Coleoptera: Scarabaeoidea) do Brasil. Monografias Tercer Milenio 1:183-195.

VAZ-DE-MELLO, F. Z. et al. 2011. Os besouros rola-bosta (Insecta: Coleoptera: Scarabaeidae: Scarabaeinae) da fazenda São Nicolau. In: Rodrigues, D. de J.; Izzo, T. J.; Battirola, L. D. (orgs.). Descobrindo a Amazônia Meridional: biodiversidade da fazenda São Nicolau. Cuiabá, MT. Editora Pau e Prosa Comunicação Ltda. pp.77-102.

VULINEC, K. 2002. Dung beetle communities and seed dispersal in primary forest and disturbed land in Amazonian. Biotropica 34(2):297-309.

WINK, C. et al. 2005. Insetos edáficos como indicadores da qualidade ambiental. Revista de Ciências Agroveterinárias (Brasil) 4(1):60-71. 